\title{
La estrategia y los sistemas integrados de gestión en las organizaciones* $^{*}$
}

\section{The strategy and the organization's integrated management systems}

\author{
Fabio Tejada Losada** \\ Universidad Santo Tomás
}

\section{RESUMEN}

Los resultados que la organización planifica alcanzar son el dinamizador de su actividad organizacional. Resultados de diferente índole que están articulados con sus propósitos vitales, ubicados dentro de la plataforma estratégica que la organización estructura con la clara intención de lograrlos. Estos propósitos, en la mayoría de los casos, están asociados a la rentabilidad, el crecimiento y la sostenibilidad.

Los sistemas de gestión -en su mayoría normalisados- resultan ser muy importantes para darle sentido
Recibido: 26 de julio de 2013

Revisado: 12 de agosto de 2013 Aceptado: 09 de septiembre de 2013 al derrotero estratégico determinado. Cuando varios de ellos confluyen, su integración es un factor que simplifica la gestión, reduce costos y hace más útil la aplicación de los sistemas como metodologías apropiadas, bajo condiciones controladas, que produce efectos favorables sobre la eficiencia y eficacia organizacional.

El punto crítico que se analiza aquí es la forma poco apropiada en que las organizaciones esperan alcanzar resultados, con ignorancia y ligereza en el abordaje de este marco de referencia. En consecuencia, lo que sería una decisión de alto impacto para fortalecer su capacidad y avanzar hacia los resultados, termina

\footnotetext{
* Artículo de revisión.

** Correspondencia: Fabio Tejada Losada. Ingeniero Industrial, especialista en Relaciones Industriales y en Administración de Empresas de la Universidad del Rosario, magíster en Calidad y Gestión Integral. Correo electrónico: fatelo@etb.net.co; fatejada07@gmail.com
} 
convirtiéndose en obstáculos que generan barreras adicionales, las cuales consumen recursos, emergiendo una gestión improductiva que debilita la dinámica de la organización.

Palabras clave: estrategia organizacional, sistemas integrados de gestión, sistemas de gestión.

\section{ABSTRACT}

The results that the organization plans to achieve are the catalyst for organizational activity. Results of different nature which are articulated with vital purposes within the organization strategic platform structure with the clear intention to achieve these vital purposes. These vital purposes in most cases are associated with the profitability, growth and sustainability.

Management systems -mostly standard- are very important to make sense of certain strategic Pilot and integration when several of them converge, is a factor that simplifies management, reducing costs and making it useful to apply the systems as appropriate methodologies under controlled conditions, which produces favorable effects on organizational efficiency and effectiveness.

The critical point to be discussed here is the inappropriately in that organizations expect to achieve results with ignorance and lightness in addressing this framework and therefore a decision which would be high impact on their ability to advance the results, is becoming obstacles created additional barriers emerging resource-consuming unproductive management undermines organizational dynamics.

Keywords: Strategy, management systems, integrated management systems.

\section{EL CUESTIONAMIENTO}

Siempre se ha reconocido que uno de los principales desafíos de todo tipo de organizaciones es alcanzar los resultados que se planifican generalmente desde los cuerpos directivos. La claridad en cuanto a la determinación y consecuente focalización en los resultados es un aspecto crucial para el desempeño de todas las organizaciones.

Un factor de alta criticidad es la forma que las organizaciones determinan para avanzar, de manera menos incierta, y alcanzar los fines planificados.

En una economía cada vez más amplia, con un sinnúmero de variables críticas que se deben advertir y gestionar para determinar el norte a seguir y no extraviar luego el rumbo definido, es necesario que las organizaciones demanden mayor juicio, mayor rigor, pero también una buena dosis de flexibilidad respecto a los análisis y la toma de decisiones apropiadas de acuerdo con los contextos en los cuales se desarrolla el quehacer empresarial.

Permanentes desafíos y constantes nuevos retos que provienen des diferentes frentes exigen de las organizaciones el desarrollo de mayor capacidad ${ }^{1}$ para trabajar de manera más eficaz y eficiente en procura de los resultados planificados. Surge aquí un primer interrogante que debe ser claramente respondido: ¿¿cuál es el tipo de resultados que busca la organización?

1 El término "capacidad" está directamente asociado a la habilidad de una organización para articular con precisión los diferentes componentes de la gestión que le permiten determinar con claridad el derrotero que se va a seguir, la toma de decisiones apropiadas y la utilización eficiente de los diferentes recursos para la realización de sus actividades, y alcanzar así los resultados planificados, con un enfoque de sostenibilidad. 
Generalmente, las organizaciones se asocian a resultados de tipo económico ${ }^{2}$, y desde tal óptica conciben todos los planes y actividades vitales para su avance. Esto, en mi concepto, es una forma de ver la situación desde el ámbito organizacional que pudiera calificarse como aceptable, pues, desde el logro alrededor de lo económico, se asegura de cierta manera la supervivencia y que la organización tenga posibilidades de -por lo menos- continuar existiendo en el entorno en el cual desarrolla sus actividades.

No hay mayor discusión en que los resultados económicos son un imperativo en las organizaciones de negocios y con ánimo de lucro. Así mismo, este tipo de logros son de suma importancia para las instituciones públicas y organizaciones de beneficencia sin ánimo de lucro; que su objetivo primordial no sea el de producir un diferencial monetario a su favor, ellas, sin lugar a dudas, deben cuidar todos los asuntos relacionados con los recursos económicos con los cuales cumplen la misión que se trazan en el presente y que les permite avanzar hacia el futuro.

En este marco de referencia, que a todas luces aparece estrecho, las organizaciones despliegan y guían toda su actividad, generando algún grado de conciencia sobre el resultado económico alrededor del cual esperan que todos los actores, desde los diferentes frentes que la componen, desarrollen y aporten lo mejor de cada uno de ellos para el logro de estas metas.

El problema comienza cuando la extrema focalización en el resultado económico, articulada de manera directa con los presupuestos establecidos, no genera mayor claridad ni despierta el entusiasmo vinculante de todos los que deben trabajar en producirlo, que debería ser el conjunto organizacional como un todo. Es necesario que, a la par con la definición de los resultados de

2 MARIÑO NAVARRETE, Hernando. Calidad: lecciones aprendidas. Bogotá: Alfaomega, 2002. 55 p. orden económico, también se determinen con claridad y establezcan en la realidad de las organizaciones otro tipo de resultados que, aun sin estar en la esencia de los logros financieros, construyan significado en el tejido de la organización, mejoren su capacidad y sirvan de fuertes palancas para precisamente alcanzar logros de tipo económico.

No debiera ser ajeno entonces a la planeación de las organizaciones, que los resultados deben ser definidos en un espectro más amplio, quedar puntualmente establecidos y alrededor de ellos generar amplia conciencia, que es otro gran reto, para concitar el interés de todos los interesados y trabajar con entusiasmo para su alcance.

Logros en el campo de la tecnología, la investigación, el fortalecimiento de los equipos de trabajo, los consecuentes métodos de trabajo, la generación de conocimiento útil para los fines establecidos, una estructura ágil y flexible -apropiada a los intereses del negocio-, procesos de comunicación efectivos que cuenten con el respaldo de procesos y procedimientos eficientes y eficaces -por ejemplo, concebidos desde la óptica de las necesidades de clientes, usuarios y/o consumidores $^{3}$ - debieran ocupar la atención de quienes piensan la organización y definen su derrotero, para que se establezcan como resultados importantes, se prioricen y, por tanto, se conviertan en motores que movilicen a la organización hacia los horizontes determinados.

\section{El camino hacia la claridad}

La estrategia es uno de los métodos que ha demostrado ser exitoso para que las organizaciones precisen

3 Generalmente, las empresas estructuran sus procesos y procedimientos desde la pura óptica interna, buscando satisfacer necesidades de control y expectativas del entorno interno. Olvidan los procesos y procedimientos que se realizan con un enfoque que permite dar respuestas a las necesidades de los clientes, usuarios y al mercado en general, para ser más eficaces y competitivos. 
con mayor claridad y focalicen su quehacer en aquellos aspectos que, como lo plantea Stephen Covey, son los "crucialmente importantes"4. Las estrategias resultan ser los medios por los cuales se logran los objetivos a largo plazo $0^{5}$. A través de la estrategia y su despliegue a proyectos, planes, tácticas, planes operativos, iniciativas estratégicas, objetivos y métodos de medición y seguimiento, la organización determina un marco de referencia para la acción y la toma de decisiones, en el cual los cuerpos gerenciales focalizan y priorizan el quehacer presente de la organización y la trayectoria que se debe seguir para avanzar hacia el futuro visionado. Todo lo anterior, por supuesto, está íntimamente asociado con los resultados, que se deben alcanzar en el corto, mediano y largo plazo.

Existen diversos métodos para la determinación de la estrategia y diferentes posturas ante esta; pudiera decirse, sin temor a equivocaciones, que se encuentran tantas concepciones como tratadistas sobre el tema. Por lo tanto, la fundamentación sobre el significado real de la estrategia debe darse en el contexto de la organización que busca utilizarla para movilizarse en un ambiente de menor incertidumbre y complejidad.

Desde este punto de vista, y como ocurre en muchos aspectos de la gestión, no hay fórmulas ni recetas que puedan ser aplicadas como tales. En cambio, sí existen métodos y procesos de investigación que deben surtirse con rigor para contar con la información veraz y pertinente, que en un ambiente de análisis que ha de involucrar los diferentes riesgos, permita tomar las decisiones indispensables para asegurar el éxito de la organización al establecer con claridad la dirección que se ha de seguir, la priorización de planes, la gestión de los recursos, la focalización en iniciativas y acciones

4 COVEY, Stephen y LINK, Greg. Smart trust. New York: Free Press, 2012. p. 29.

5 DAVID, Fred R. Conceptos de administración estratégica. México: Pearson Educación, 2003. p. 368. coherentes para alcanzar los resultados, así como también el control necesario para monitorear el desempeño de la empresa.

Se requiere, pues, trabajar en la construcción y el fortalecimiento de una plataforma estratégica que dé claridad y sentido respecto a aquellos aspectos que la organización se propone lograr. Esta plataforma parte de la definición de la misión de la empresa, que se recomienda construir con la participación de las personas 0 , en el peor de los casos, si las gentes de la organización no han participado en su definición, ser desplegada a todos los niveles, buscando su entendimiento, aceptación y reconocimiento por parte de los diferentes actores de la organización. La misión debe traducir en lenguaje sencillo la razón de ser de la organización; por qué y para qué existe; los productos, servicios, mercados y soluciones que produce; y debe describir aquello que la organización es en el presente. Diversos autores determinan la misión como "el credo empresarial".

Los valores institucionales conforman otro componente de alta importancia que permite fortalecer y dar sentido a la plataforma estratégica. Como ocurre con la misión, los valores han de establecerse con la participación de las personas, a partir del conjunto de creencias que proviene del colectivo; por ende, están influenciados por los estilos de dirección y por las actitudes de los cuerpos directivos. Estos conjuntos de creencias guían el comportamiento de todos sus miembros y dan las pautas precisas de cómo comportarse dentro y fuera de la organización. Los valores determinan lo importante en lo que creen firmemente las personas, regulan la vida de una organización y, por tanto, deben ser compartidos por todos, pues definen la actuación de las personas dentro de la organización y hacia sus clientes externos. 
La visión, concebida como la concepción amplia del futuro de una organización, permite visualizar con claridad hacia dónde se dirige la empresa, pues describe el marco de referencia que la organización determina como alcanzable y ambicioso, pero realizable, que permite direccionar los esfuerzos de todas las partes interesadas para alcanzar una situación mejor que la presente. Por medio de la visión se buscan mejores resultados para la empresa, en una clara articulación con la atención y la satisfacción de mercados ampliados en los cuales se espera que los retornos y las retribuciones a todos los grupos de interés se incrementen y, por lo tanto, resulten de alto beneficio. La visión así descrita y compartida, robustece la plataforma estratégica para asociar esfuerzos de todas las personas hacia un propósito común dentro de un claro enfoque sistémico.

Los tres pilares anteriores -misión, visión y valoresconstituyen un referencial determinante para la adopción de la(s) estrategia(s) que, como quedó establecido anteriormente, permite(n) focalizar las iniciativas de la empresa para alcanzar los grandes propósitos de la organización -resultados- y la definición de metas y objetivos con mayor precisión y sin ambigüedades.

El análisis juicioso del entorno interno y externo en que la empresa se desempeña es determinante para que la organización descubra y precise las oportunidades que el mercado ofrece, y en una clara articulación con sus posibilidades, defina el trayecto por seguir.

La estrategia debe producir y transmitir claridad sobre cómo se va a alcanzar la misión y la visión de la organización, afianzada esta en un conjunto de valores. Debe ser clara en trasmitir con precisión y sencillez el sentido de propósito de la organización.

El despliegue de la estrategia a las unidades de negocio o de trabajo, procesos, áreas o divisiones de la organización se materializa mediante los planes operativos o tácticos, que permiten pasar de la planeación a la ejecución ${ }^{6}$; son la articulación precisa de los aspectos planeados para el largo plazo, con el corto plazo, con el día a día de la organización. Objetivos y metas de tipo estratégico constituyen el tejido que amarra la(s) estrategia(s) en las actividades que la organización debe realizar prioritariamente para alcanzar los resultados estratégicos del negocio.

La consecución, distribución y uso apropiado de recursos le dan 'oxígeno' a la organización para hacer realidad la estrategia. Con frecuencia se plantea que una vez definidos los derroteros estratégicos para la empresa y sus consecuentes planes para la acción, no debiera destinarse recurso organizacional alguno que no esté estrechamente articulado con los aspectos estratégicos definidos.

La eficiencia para alcanzar el resultado, la eficacia para la concentración y el trabajo enfocado en los objetivos planificados son temas de alta criticidad que aseguran el logro de los resultados estratégicos, que debiera conducir a la organización a contar con personas motivadas y preparadas, procesos eficientes y eficaces, sistemas de trabajo efectivos, clientes con sus necesidades y expectativas satisfechas por medio de los productos y/o servicios que reciben, dispuestos a repetir experiencias de compra con la organización, y accionistas satisfechos con los resultados y rendimientos obtenidos?

6 Diferentes tratadistas plantean que el verdadero reto para las organizaciones está en llevar a la acción los diferentes aspectos planificados. Michael Porter sostiene que la aplicación de la estrategia demanda de un líder estratega que abandere con entusiasmo y decisión las estrategias definidas para el negocio -desde el enfoque de la generación compartida de valor-, asegurando que se repitan a lo largo y ancho de la organización las iniciativas estratégicas, pues aquello que no se hace constante y consistentemente no es estratégico para la empresa y, por tanto, no produce ninguna clase de diferenciación.

7 Kaplan y Norton, en su modelo para el control de la estrategia (Balanced Score Card) proponen este esquema, que busca, en una secuencia de causa-efecto, asociar los componentes críticos que la 
Toda esta construcción ha de generar la claridad y el enfoque suficientes para que la organización tenga altas probabilidades de vigorizar su capacidad de desempeño y la haga más efectiva en el entorno en el que actúa.

\section{La generación de capacidad}

Ocupándonos nuevamente de la capacidad de la organización, según lo ya establecido, la capacidad debiera comenzar a construirse a partir de la claridad que se genere desde la plataforma estratégica que coherentemente debe orientar a la empresa para avanzar en un ambiente de menor incertidumbre en busca del logro de sus objetivos.

En este punto surge otro desafío para la organización, pues debe ocuparse ahora de cómo lograr que lo planeado se convierta en realidad de la manera más económica. Es entonces cuando las organizaciones deben repensar el método o los métodos más apropiados para producir los resultados esperados en un escenario como el antes descrito. Es bien sabido que lo que se planifica no resulta idéntico en la práctica al ejecutarlo; se presentan variaciones que dependen de muchos factores: uno de ellos es la forma en que buscamos poner en acción los aspectos planificados. En esta forma -que aquí denomino como métodos de trabajo-los factores humanos y la tecnología que usa la organización en sus procesos de conversión y de la prestación del servicio tienen gran influencia.

El reto para cualquier tipo de organización está en que el costo que le significa producir productos y/o servicios -llevarlos a un mercado, darlos a conocer para que sean

organización considera para gestionar la estrategia, y plantean para ello cuatro perspectivas: la del aprendizaje y el conocimiento, la perspectiva interna de los procesos, la externa de los clientes y la de los accionistas. Para la generación de valor, consideran que se deben vincular al proceso los "activos intangibles", propios de la era del conocimiento, para que, actuando en conjunto con los activos tangibles, la organización tenga mayores posibilidades de alcanzar los resultados estratégicos planificados. adquiridos por clientes/usuarios-sea menor que el valor económico que retorna a la organización como resultado de este ejercicio o, en el peor de los casos -a revertir a favor de la organización- que por lo menos sea igual'. Si la organización mantiene el resultado de esta ecuación a su favor, desarrollando métodos que lo hagan posible en el tiempo, existen altas probabilidades, por lo menos desde este punto de vista, de perdurar, crecer, innovar, de ser responsable socialmente -siéndolo primero con sus colaboradores-, de trabajar en mantener un equilibrio ambiental y asegurar así un establecimiento más sólido en y para con todos los grupos de interés.

Los colaboradores de la organización, en todos los niveles, son lo que llevan a la práctica y hacen posible o no que los diferentes aspectos planificados para los procesos, productos/servicios, unidades de trabajo, unidades de negocio se conviertan en realidad y se produzcan los resultados esperados. En consecuencia, debe generarse la suficiente claridad en el sentido de propósito, para que el trabajo que se realiza en el día a día de la empresa se asocie de manera estrecha con los diferentes aspectos que han quedado planificados. Aquí los métodos que la organización establece y adopta -la claridad, decisión y vocación con que lo hace- se vuelven determinantes para aclarar el escenario para la acción 0 para complicar la vía por la cual han de circular los diferentes componentes de la organización, integrándose y articulándose coherentemente para producir los efectos deseados en todos los frentes y procesos de trabajo que, en sumatoria, deben fortalecer la capacidad para alcanzar los resultados.

En otros términos, dirección con enfoque, personas con esa claridad de enfoque, competencias necesarias, tecnología adecuada y métodos de trabajo apropiados,

8 En ocasiones que se asocian a estrategias de penetración en un nuevo mercado o productos nuevos, se invierte esta ecuación, pero por tiempos claramente calculados, para producir los efectos esperados en la captura de mercados o posicionamiento de productos. 
entre otros no menos importantes ${ }^{9}$ son factores vitales para que los aspectos planificados pasen a la acción y busquen convertirse en realidades para la organización.

\section{La decisión inteligente}

Los sistemas de gestión normalizados surgen en estos escenarios como apoyos extraordinarios que permiten dinamizar de mejor forma la acción de la organización, según sus necesidades, al entregarles a las personas de todos sus niveles orientaciones que constituyen lo que aquí se ha denominado como métodos de trabajo apropiados, para que dentro de la claridad de enfoque construido se logre pasar del pensamiento a la acción productiva.

El sistema de gestión de la calidad, -establecido con base en la filosofía, las orientaciones y los requisitos de la familia de normas ISO 9000 que buscan darle asistencia a la organización (métodos apropiados) para trabajar con eficacia y eficiencia centrada en la satisfacción de sus clientes-, enfoca la organización a la efectividad en la búsqueda de los resultados, al minimizar los riesgos -en su entorno externo e interno- de no cumplir con los requisitos, las necesidades y expectativas de sus clientes, que se materializan en productos y servicios, así como de los requisitos legales y reglamentarios.

En la introducción de la NTC ISO 9000/2005, publicada por el ICONTEC Internacional, se expresa textualmente lo siguiente:

\section{La familia de Normas ISO 9000 citadas a continuación} se han elaborado para asistir a las organizaciones, de

9 Es necesario precisar que la generación de un clima apropiado para la acción, que se deriva de los estilos adecuados de dirección, la comunicación asertiva y oportuna, el conocimiento requerido, los recursos necesarios disponibles que posibilitan una mayor motivación del personal son elementos que no pueden estar ausentes en el ámbito de las organizaciones para fortalecer las probabilidades de avanzar con menos dificultades hacia los objetivos planificados. todo tipo y tamaño, en la implementación y la operaciónde sistemas de gestión de la calidad eficaces.

- La Norma ISO 9000 describe los fundamentos de los sistemas de gestión de la calidad y especifica la terminología de los sistemas de gestión de la calidad.

- La Norma ISO 9001 especifica los requisitos para los sistemas de gestión de la calidad aplicables a toda organización que necesite demostrar su capacidad para proporcionar productos que cumplan los requisitos de sus clientes y los reglamentarios que le sean de aplicación y su objetivo es aumentar la satisfacción del cliente.

- La Norma ISO 9004 proporciona directrices que consideran tanto la eficacia como la eficiencia del sistema de gestión de la calidad. El objetivo de esta norma es la mejora del desempeño de la organización y la satisfacción de los clientes y de las partes interesadas.

- La Norma ISO 19011 proporciona orientación relativa a las auditorías de sistemas de gestión de la calidad y de gestión ambiental.

Todas estas normas juntas forman un conjunto coherente de normas de sistemas de gestión de la calidad que facilitan la mutua comprensión en el comercio nacional e internaciona ${ }^{10}$.

Para el sector público se adecuó la norma NTCGP 1000, que se encuentra en versión del año 2009 y que recoge las orientaciones y los requisitos de la NTC ISO 9000, 9001 y 9004. Esta versión se presenta de manera simultánea con el MECI (método estándar de control interno para el Estado colombiano).

10 INSTITUTO COLOMBIANO DE NORMAS TÉCNICAS Y CERTIFICACIÓN. Sistemas de gestión de la calidad. Fundamentos y vocabulario. NTC ISO 9000:2005. Bogotá D.C.: El Instituto, 2005. $36 \mathrm{p}$. 
El sistema de gestión ambiental, orientado por la familia de normas ISO 14000 (ISO 14001), se estableció para dotar a las organizaciones de cualquier naturaleza que están comprometidas con asegurar un desempeño apropiado en materia del control de los impactos que produce en el medio ambiente, con ocasión de la realización de productos y/o servicios.

Organizaciones de todo tipo están cada vez más interesadas en alcanzar y demostrar un sólido desempeño ambiental mediante el control de los impactos de sus actividades, productos y servicios sobre el medio ambiente, acorde con su política y objetivos ambientales. Lo hacen en el contexto de una legislación cada vez más exigente del desarrollo de políticas económicas y otras medidas para fomentar la protección ambiental y de un aumento de la preocupación expresada por las partes interesadas por los temas ambientales, incluido el desarrollo sostenible ${ }^{11}$.

El sistema de gestión de seguridad y salud ocupacional, desarrollado por el conjunto de normas OHSAS 18000 (OHSAS 18001), busca dotar a la organización de un sistema de gestión de prevención de riesgos laborales, con el fin de garantizar ambientes de trabajo más seguros, que no afecten a las personas en su integridad y, en consecuencia, que las diferentes labores que una organización adelanta para cumplir con sus diferentes procesos no se entorpezcan.

La serie de normas OHSAS 18000 están planteadas como un sistema que dicta una serie de requisitos para implementar un sistema de gestión de salud y seguridad ocupacional, habilitando a una empresa para formular una política y objetivos específicos asociados al tema, considerando requisitos legales e información sobre los riesgos inherentes a su actividad. Estas

11 INSTITUTO COLOMBIANO DE NORMAS TÉCNICAS Y CERTIFICACIÓN. Sistemas de gestión ambiental. Requisitos con orientación para su uso. NTG ISO 14001:2004. Bogotá D.C.: El Instituto, 2004. 28 p. normas buscan asegurar el mejoramiento de la salud y seguridad en el lugar de trabajo mediante una gestión sistemática y estructurada ${ }^{12}$.

Todas las anteriores normas son compatibles, lo cual facilita una gestión conjunta a cualquier tipo de organizaciones.

El sistema de gestión de riesgos, orientado por la norma "ISO 31000 para la gestión del riesgo. Principios y directrices", da orientación para que la organización sea más eficaz en el tratamiento de factores que la exponen a incertidumbres en su entorno externo e interno para el logro de sus objetivos.

En la introducción de la NTC ISO 31000/2011, publicada por el ICONTEC Internacional, se expone puntualmente lo siguiente: "Esta norma recomienda que las organizaciones desarrollen, implementen y mejoren continuamente un marco de referencia cuyo propósito sea integrar el proceso para la gestión del riesgo en los procesos globales de gobierno, estrategia y planificación, gestión, procesos de presentación de informes, políticas, valores y cultura de la organización. Todas las actividades de una organización implican riesgo. Las organizaciones gestionan el riesgo mediante su identificación y análisis y luego evaluando si el riesgo se debería modificar por medio del tratamiento del riesgo con el fin de satisfacer los criterios del riesgo" ${ }^{13}$.

Las anteriores normas -recogiendo en este rápido análisis las más aplicadas por la organizaciones en nuestro medio- constituyen un excelente marco de referencia para que las organizaciones de diferente naturaleza

12 CEPYME ARAGÓN. Procedimientos basados en las normas OSHAS 18000 para su implantación en pymes del subsector fabricación de productos metálicos. Zaragoza: Confederación de la Pequeña y Mediana Empresa Aragonesa, 2003. p. 207.

13 INSTITUTO COLOMBIANO DE NORMAS TÉCNICAS Y CERTIFICACIÓN. Gestión del riesgo. Principios y directrices. NTC ISO 31000:2011. Bogotá D.C.: El Instituto, 2011. p. 31. 
construyan un ordenamiento superior que les asegure una actuación focalizada y bajo condiciones controladas para alcanzar los resultados planificados dentro de sus propósitos fundamentales, que resultan ser los estratégicos para las empresas. En otros términos, estos sistemas normalizados debieran potenciar la capacidad de la organización como un todo para que los resultados se alcancen dentro de un contexto de alta eficiencia y eficacia, llevándola a una mejora sostenida de su desempeño.

El problema mayúsculo aquí es la forma en que las organizaciones abordan estos modelos normalizados; generalmente lo hacen por presión de los proveedores, por imitación, porque la competencia u otras organizaciones los aplican o porque a alguien de la Dirección le parece interesante. Esta aproximación a los sistemas normalizados es desastrosa, pues no suma valor a la organización en la mejora de los procesos, no genera claridad ni fluidez en las actividades a desarrollar, sino que, por el contrario, produce mayor confusión y congestión en el tránsito que la organización debe realizar para alcanzar sus objetivos.

Una decisión inteligente estaría determinada por el claro enfoque estratégico de la dirección de la organización, que la llevaría resueltamente a adoptar los sistemas de gestión -normalizados o no- que estén claramente articulados con su plataforma estratégica y profundamente relacionados con las estrategias y actividades estratégicas que va a desarrollar para alcanzar los resultados planificados.

Por tanto, la claridad del enfoque estratégico de la organización es determinante para la adopción de cualquier tipo de sistema de gestión. Plantearlo así suena simplista y hasta reduccionista; pero la realidad nos muestra permanentemente que en no pocos casos, organizaciones de diferente naturaleza y tamaño buscan desarrollar sistemas de gestión sin una clara articulación con sus propósitos vitales.
En consecuencia, no debiera tratarse de establecer sistema de gestión alguno si este no está estrechamente articulado con el desarrollo de capacidad organizacional para responder a los diferentes retos estratégicos y si no hace posible alcanzar los resultados de manera efectiva.

Otro aspecto no menos crítico en algunas organizaciones es la proliferación de sistemas que parecen ir cada uno por su lado determinado, básicamente por el cumplimiento de una serie de requisitos. Esto consume demasiada energía de las personas y no se traduce en resultados eficaces frente a los propósitos vitales de la organización.

Al no estar cimentados los sistemas de gestión a partir de las necesidades estratégicas de las empresas, la falta de claridad hace que caigan fácilmente en el afán de responder a los requisitos de las normas que orientan los sistemas, sin un sentido práctico y de articulación con los propósitos fundamentales derivados de sus plataformas estratégicas. Es decir, el requisito se vuelve el fin en sí mismo y no en el medio apropiado que la organización debe aplicar para alcanzar el objetivo propuesto, dando como resultado que el(los) sistema(s) se convierta(n) en la finalidad y no en el medio metodológico apropiado para fortalecer la dinámica de la mejora de la organización y lograr sosteniblemente sus resultados vitales.

Como quedó dicho y se reitera, la decisión inteligente pasa por un consciente análisis de las demandas de renovada capacidad de la organización para enfrentar los desafíos estratégicos y las exigencias legales, capacidad que la lleve a la adopción de sistemas de gestión -métodos de trabajo bajo condiciones controladas en el ciclo de la calidad- ${ }^{14}$ y que le permitan lograr sus resultados fundamentales de una forma sostenible.

14 En la filosofía general que orienta los diferentes sistemas de gestión, es claro que ellos se deben establecer en el ciclo PHVA (planear, hacer, verificar, actuar) como marco de referencia apropiado. 
Aparejada a la anterior, otra decisión inteligente es la visualización de la dirección de la organización para que, dentro de un enfoque sistémico, conciba como un todo los sistemas necesarios para el fortalecimiento de la capacidad organizacional, en un ambiente de integración ${ }^{15}$ de tales sistemas y de la integralidad de la gestión de la empresa.

Para la integración de los diferentes componentes de los sistemas, es necesario recurrir nuevamente al marco estratégico y a los objetivos de la organización, y conocer cuáles de ellos son las necesidades de, por ejemplo, precisión de enfoque en el cliente (requisitos y expectativas de los clientes), determinación y tratamiento de peligros en el campo de la seguridad industrial y salud ocupacional de los trabajadores, de las exigencias resultado de los análisis ambientales para la organización (análisis de aspectos ambientales significativos) y de los riesgos de diferente orden que la empresa enfrenta (determinación de contextos y enfoques del riesgo).Todo lo anterior es necesario porque apalanca de manea crítica y contundente las actividades estratégicas de la organización. En consecuencia,

15 Conforme las empresas van definiendo e implantando sistemas de gestión, en su mayoría con vocación, certificables, se hace más evidente la necesidad de racionalizar los esfuerzos, costos y recursos destinados a estos. Sobre todo cuando las normas de referencia en las que se basan comparten requisitos en un porcentaje importante, y la metodología de gestión es al 100 \% idéntica. Por lo tanto, el planteamiento de optimizar recursos, costos y esfuerzos vendrá por la integración común de todos aquellos conceptos cuya gestión estratégica tienen aspectos y requisitos comunes. El objetivo no es otro que evitar duplicidades, optimizar recursos y simplificar al máximo la gestión de todos los sistemas, haciéndolos visibles en su utilidad práctica para alcanaar los resultados.

Por tanto los ambientes de integración están concebidos como el proceso a través del cual la organización aprende a introducir criterios y especificaciones en sus procesos y en sus sistemas de modo que satisfagan a todos sus clientes (internos, externos, institucionales, partes interesadas, etc.) de forma simultánea, ahorrando costos y esfuerzos, con un espíritu innovador, autocrítico y comprometido con la mejora continua, dentro y para el marco estratégico definido por la empresa (Información tomada del portal http:/lexcelencia-empresarial. com/sistemas_gestion.htm, mejorada por el autor). se debe trabajar en estos sistemas con claros criterios de utilidad práctica y de frente a los resultados esperados. Desde este enfoque debe surgir la visión integradora de los sistemas.

Infortunadamente, se ha evidenciado que la aproximación que se busca de la integración con frecuencia está conducida por una perspectiva asociada únicamente al cumplimiento per se de los requisitos de las normas y de manera desarticulada de los propósitos vitales de la organización, lo que constituye un verdadero desperdicio de energía organizacional.

Tal aproximación desencadena otro escenario indeseable: los sistemas normalizados se vuelven perceptibles solo desde el punto de vista documental; en consecuencia, en el imaginario de la organización se trabaja dando prioridad y énfasis extremo a todos los requisitos de orden documental, lo que produce una verdadera intoxicación de documentos y registros que en la mayoría de los casos no son necesarios.

Desde otra perspectiva, pero dentro del mismo marco de análisis, es poco inteligente y productivo para la organización tratar de buscar la integración de los sistemas solamente a partir de la asociación de sus requisitos comunes, trasladando todo este esfuerzo de comparación al campo documental, sin considerar el horizonte estratégico de la empresa en primer término y de manera crucial como base fundamental y, por tanto la utilidad, que le aportaría uno u otro sistema para avanzar hacia el horizonte estratégico definido y buscar la determinación de sistemas útiles para, desde esta perspectiva, producir su integración.

Esta decisión inteligente, por supuesto, debe tener como desenlace la precisión de las metodologías y la claridad de la organización, que debe fortalecer su capacidad para alcanzar los resultados estratégicos de manera sostenible. 


\section{REFERENCIAS}

CEPYME ARAGÓN. Procedimientos basados en las normas OSHAS 18000 para su implantación en pymes del subsector fabricación de productos metálicos. Zaragoza: Confederación de la Pequeña y Mediana Empresa Aragonesa, 2003. 207 p.

COVEY, Stephen y LINK, Greg. Smart trust. New York: Free Press, 2012. 320 p.

DAVID, Fred R. Conceptos de administración estratégica. México: Pearson Educación, 2003. 368 p.

INSTITUTO COLOMBIANO DE NORMAS TÉCNICAS Y CERTIFICACIÓN. Sistemas de gestión de la calidad. Fundamentos y vocabulario. NTC ISO 9000:2005. Bogotá D.C.: El Instituto, 2005.36 p.
INSTITUTO COLOMBIANO DE NORMAS TÉGNICAS Y CERTIFICACIÓN. Sistemas de gestión ambiental. Requisitos con orientación para su uso. NTC ISO 14001:2004. Bogotá D.C.: El Instituto, 2004. $28 \mathrm{p}$.

INSTITUTO COLOMBIANO DE NORMAS TÉCNICAS Y CERTIFICACIÓN. Gestión del riesgo. Principios y directrices. NTC ISO 31000:2011. Bogotá D.C.: El Instituto, 2011.31 p.

KAPLAN, Robert y NORTON, David. La Organización focalizada en la Estrategia. Barcelona: Ediciones Gestión, 2005, 2001. 424 p.

MARIÑO NAVARRETE, Hernando. Calidad: lecciones aprendidas. Bogotá: Alfaomega, 2002. 55 p.

PORTER, Michael. Ser competitivo. Nuevas aportaciones y conclusiones. Bilbao: Ediciones Deusto, 1999. 478 p. 